\title{
Penile Compression Clamps: a model of the internal mechanical state of penile soft tissues
}

\begin{tabular}{|r|l|}
\hline Journal: & Neurourology and Urodynamics \\
\hline Manuscript ID & NAU-16-0334 \\
\hline Wiley - Manuscript type: & Original Clinical Article \\
\hline Subject Sections: & Bioengineering, Incontinence (male), Neurourology: Clinical \\
\hline Keywords: & Biomechanical modeling, Finite element simulations, penile clamps \\
\hline \multicolumn{2}{|}{} \\
\hline
\end{tabular}

\section{SCHOLARONE ${ }^{m}$ \\ Manuscripts}




\begin{abstract}
Aims: Prostate cancer is the most frequently diagnosed cancer, and male incontinence represents a major consequence following surgery. Penile compression clamps (PCCs) which externally occlude the urethra, may be used to manage the incontinence. Despite potential complication of PCCs, such as deformation-inflicted tissue damage, to date, there are no reported biomechanical criteria for design of PCCs, in terms of quantitative parameters for evaluating the safety-versus-efficacy of existing or future designs.

Methods: we developed a set of computational three-dimensional models of the penis, to which compression was applied using five generic PCC designs. The internal mechanic states of the soft tissues of the penis were then analysed using finite element simulations.

Results: Stresses in skin, fat and tunica albuginea regularly exceeded $10 \mathrm{kPa}$ $(75 \mathrm{mmHg})$. Cuff-type and knurl-type PCCs pose the highest potential risks to tissue health with elevated tissue stresses around the entire penile perimeter (cuff) or urethral stress concentrations (knurl). The soft and contoured PCCs produced the lowest values of these mechanical parameters.

Conclusion: The present study identified design characteristics, being envelopment, adaptability and durability, which provide the safest mechanical conditions in the penis and thus minimize the risk of tissue damage while still managing incontinence. Such data should help to design a safer clamp. Frequent off-loading of the penile tissues should be emphasized to patients, to prevent deformation-inflicted tissue damage.
\end{abstract}




\section{Introduction}

Among the many types of cancer, prostate cancer is the most frequently detected, with an estimated 220,800 men diagnosed in 2015 , accounting for $26 \%$ of all new cancer cases in $2015 .^{1}$ The primary treatment options include radical prostatectomy and external beam radiation. ${ }^{2}$ However, urinary incontinence represents a common complication following these procedures, with incidence ranging widely between $1 \%$ and $47 \%{ }^{3}$ Indeed, a US population-based study in 2000 reported that $40.2 \%$ of the patients experienced occasional urinary leakage, $6.8 \%$ experienced frequent urinary leakage and $1.6 \%$ suffered no urinary control in the period $18-24$ months after surgery. ${ }^{2}$

To control leakage, men resort to absorbent pads, urinary sheaths, body-worn urinals and penile compression clamps (PCCs). ${ }^{4,5}$ Designed to externally occlude the urethra, PCCs have been reported to reduce the impact of incontinence and be particularly useful for managing incontinence during activities. ${ }^{6,7}$ However, PCCs do not completely eliminate urine leakage when applied at a comfortable pressure and pain is reported when compression is sufficient to prevent leakage. ${ }^{5,6}$ Case reports describe reduced penile blood flow and deformation-inflicted tissue damage, but there is no evaluative data assessing the effect of various penile clamp designs and the real life potential for injury. ${ }^{8,9}$ The purpose of this study was to develop a computational model of the internal mechanical state of the penis when compressed by a range of PCC designs. This study is part of a larger programme of work to design an effective, comfortable and low risk PCC. 
1

2

3

4

5

6

7

8

9

10

11

12

13

14

15

16

17

18

19

20

21

22

23

24

25

26

27

28

29

30

31

32

33

34

35

36

37

38

39

40

41

42

43

44

45

46

47

48

49

50

51

52

53

54

55

56

57

58

59

60

John Wiley \& Sons 


\section{Materials \& Methods}

\section{Geometry}

Twelve FE model variants (models) were developed, representing five generic designs and three stiffnesses of interface materials representative of PCCs currently available on the internet (see Table 1). Each model was based on an anatomical description of the 3D structure of the penis, informed by previous studies by the authors, which included the skin, fat, tunica albuginea (TA), the corpus cavernosa (CC), corpus spongiosum (CS) and urethra (Figure 1a,b). ${ }^{10,11}$ Two horizontal cuboids with sizes of $10 \times 10 \times 50 \mathrm{~mm}$, representing a flat clamp, were added above and below the shaft of the penis in models $F 25, F 50$ and $F 100$ (Figure 1c). In models $A 25, A 50$ and $A 100$, the flat clamp was replaced with an angled clamp, which represents clamps intended for use in a bent state, to follow the contours of the penis (Figure 1c). A contoured clamp, which was based on the curved outer contour of the penis, was incorporated in models C25, C50 and C100 (Figure 1a). In Cuff model, a uniform circumferential pressure was applied over a $10 \mathrm{~mm}$-wide band, to simulate a soft cuff-type PCC. Knurl model included the top-half of a contoured clamp and a half-sphere, $20 \mathrm{~mm}$ in diameter, representing a protrusion designed to locally push against the urethra (Figure 1c). Finally, in order to simulate a situation in which the device was poorly positioned or malfunctional, we used the Tilt model, where the bottom half of a $50 \mathrm{kPa}$ flat clamp was tilted at $5^{\circ}$.

The 3D geometrical modeling was performed using ScanIP® module of the Simpleware $®$ software suite. ${ }^{12}$ The dimensions of the axial cut through the penile shaft were $32 \times 40 \mathrm{~mm}$, and the total length of the penis was $110 \mathrm{~mm}$. 


\section{Mechanical properties of the tissues and clamps}

The constitutive laws and mechanical properties of all the tissue components were adopted from the literature. Specifically, the skin and TA were considered as transverse-orthotropic-elastic materials, which obey the following constitutive law: ${ }^{13,14}$

$$
\left[\begin{array}{c}
S_{x x} \\
S_{y y} \\
S_{z z} \\
S_{x y} \\
S_{y z} \\
S_{z x}
\end{array}\right]=\left[\begin{array}{cccccc}
\frac{1-v_{y z} v_{z y}}{C_{y} C_{z} \Delta} & \frac{v_{y x}-v_{z x} v_{y z}}{C_{y} C_{z} \Delta} & \frac{v_{z x}-v_{y x} v_{z y}}{C_{y} C_{z} \Delta} & 0 & 0 & 0 \\
\frac{v_{x y}-v_{x z} v_{z y}}{C_{z} C_{x} \Delta} & \frac{1-v_{z x} v_{x z}}{C_{z} C_{x} \Delta} & \frac{v_{z y}-v_{z x} v_{x y}}{C_{z} C_{x} \Delta} & 0 & 0 & 0 \\
\frac{v_{x z}-v_{x y} v_{y z}}{C_{x} C_{y} \Delta} & \frac{v_{y z}-v_{x z} v_{y x}}{C_{x} C_{y} \Delta} & \frac{1-v_{x y} v_{y x}}{C_{x} C_{y} \Delta} & 0 & 0 & 0 \\
0 & 0 & 0 & 2 C_{x y} & 0 & 0 \\
0 & 0 & 0 & 0 & 2 C_{y z} & 0 \\
0 & 0 & 0 & 0 & 0 & C_{z x}
\end{array}\right]\left[\begin{array}{c}
E_{x x} \\
E_{y y} \\
E_{z z} \\
E_{x y} \\
E_{y z} \\
E_{z x}
\end{array}\right],
$$

where $S_{i j}$ are the components of the tissue stress tensor, $E_{i j}$ are the components of the tissue strain tensor, $C_{i}$ are the elastic moduli, $C_{i j}$ are the shear moduli, $v_{i j}$ are Poisson's ratios and

$$
\Delta=\frac{1-v_{x y} v_{y x}-v_{y z} v_{z y}-v_{z x} v_{x z}-2 v_{x y} v_{y z} v_{z x}}{C_{x} C_{y} C_{z}}
$$

The fat, CC and CS were assumed to be linear-elastic isotropic materials with elastic moduli of $80 \mathrm{kPa}$ and $20 \mathrm{kPa}$, and Poisson's ratios of 0.4 and 0.49 , respectively. ${ }^{10,11}$ The interface materials of all the PCCs were also considered isotropic linear-elastic, with elastic moduli of 25, 50 and $100 \mathrm{kPa}$, and a Poisson's ratio of 0.3 .

\section{Boundary and material transition conditions}


Boundary conditions were chosen to simulate modes of loading of the PPCs (Figure 1a). First, the proximal surfaces of skin, fat, TA, CC and CS were fixed for all motions and tied interfaces were defined between all tissue components. Then, in all models but one, opposite displacements in the range of $0.9-2.1 \mathrm{~mm}$ were prescribed to the top and bottom surfaces of the clamp, so that the penis was compressed between the two parts of each clamp (Figure 1a). In Cuff model variant, a uniform circumferential pressure of 5 $\mathrm{kPa}$ was applied in a $10 \mathrm{~mm}$-width band, corresponding to the width and position of the clamps.

\section{Development of the computational simulations}

Meshing of tissues and angled/contoured clamps was performed using the ScanIP® module of the Simpleware $₫$ software suite, with specific refinement of the CS around the urethra. ${ }^{12}$ Meshing the flat clamp and half-sphere of the knurl was performed in the Preview module of FEBio. ${ }^{13,14}$ Simulations were set up using the PreView module of FEBio (Ver. 1.16.3), analyzed using the Pardiso linear solver of FEBio (http://mrl.sci.utah.edu/software/febio) (Ver. 2.3.1) and post-processed using the PostView tool of FEBio (Ver. 1.9). ${ }^{13,14}$

\section{Outcome measures}

Effective and maximal shear strain and stress distributions were examined in penile soft tissues and compared to each model for urethral obstruction corresponding to a $50 \%$ reduction in its undeformed cross-sectional area. Numerical convergence issues involving critical element distortions prevented simulations of complete, i.e. $100 \%$ occlusions. Nevertheless, the $50 \%$-extent closure served as an objective, comparative, 
standard measure without introducing computational errors and biases related to overdistortion of individual elements. Strain and stress data were pooled separately, for each tissue type, using values from all of the elements located in the $10 \mathrm{~mm}$ strip of the deformed penile shaft area.

\section{Results}

In all models, peak effective strains occurred in the inner aspect of the CS, laterally to the obstructed urethra, with maximum effective strains of up to 0.73 . Higher strains corresponded to designs with greater material stiffness (F100, A100 and C100). Increased contact area with the penile skin, corresponding to contoured models, resulted in more uniform distributions of internal tissue strains (Table 1). Knurl model yielded the most extreme strain concentrations in the CS, fat and skin tissues near the urethra.

Effective stress distributions in soft tissues at 50\%-urethral obstruction are indicated in Figure 2. Maximal effective stresses were found in skin and fat tissues under the urethra, and stress values increased with the stiffness of the interface material in the flat, angled and contoured models. Interestingly, peak effective stresses shifted laterally with an increase in the PCC contact area (Table 1). In the stiffer model variants (F100, A100 and C100), both average and maximal skin strains and stresses generally increased for greater contact area of the clamp with the skin (Figure 3). Minimal exposures to skin strains and stresses, safer in terms of tissue health, were found in the soft angled and contoured variants $(A 25, C 25)$. In Cuff model we found increased skin stresses around the penis (elevated average skin loads) whereas in model Knurl we found concentrated increased skin stresses locally under the urethra (highest maximal 
skin loads) (Figure 3). Fat tissue exhibited loading patterns similar to those of skin tissue; however, the influence of increased contact area of the clamp with the skin was less distinct in fat (Figure 3).

Simulation of poor positioning or mal-function of the flat clamp (50kPa stiffness) resulted in a small increase in both effective strains and stresses in all soft tissues when compared to the equivalent model F50 (Figure 4). As an example, the maximal and average effective and shear strains increased by an average of $5.7 \%, 4.9 \%$ and $5.6 \%$ in the skin, fat and TA, respectively. The corresponding mean increases for maximal and average effective shear stresses were $7.2 \%$ and $1.4 \%$ in skin and fat tissues, respectively, although there were negligible changes in the TA.

\section{Discussion}

To date, there are no reported biomechanical criteria for PCCs related to quantitative parameters for evaluating the safety-versus-efficacy of existing or future designs. The present study was therefore intended to identify specific characteristics to provide the safest mechanical conditions in the penis, and thus minimize the risk of tissue damage while still providing continence. Twelve FE model variants were used, incorporating five PCC designs. The variants accommodated factors such as the interface material stiffness, the contact area of the clamp with the skin and the nature of use of the device.

Overall, peak and average effective stresses in the TA were in good agreement with maximal stress data associated with normal erection, and average effective stresses following implantation of an inflatable penile prosthesis. ${ }^{10,11}$ Our findings indicated that increasing the stiffness of interface materials inflicted greater strains and stresses in the 
tissues and that PCCs with relatively stiff interface materials were unable to cushion the soft tissues of the penis while applying external compression to constrict the urethra; PCCs with softer interface materials were able to reduce peak tissue strains and stresses and achieve more uniform strain and stress distributions in the soft tissues (Figures 2). Additionally, an increased contact area with the skin of the penis, as achieved with the contoured and angled PCCs (Table 1), allowed for increased area for load transfer and more uniform distributions of internal tissue loads.

Our results indicate that the mechanism of closure for cuff-type PCCs is less efficient from a mechanical perspective. This may be attributed to the elliptical cross-section of the unloaded urethra (Figure 1b), which collapses more easily under vertical compression than under circumferential pressure. Nevertheless, directly-targeted compression against the urethra from below, applied locally via a protrusion as examined in the Knurl model variant, is also not recommended. The resulting concentrated strains and stresses in both the fat and skin tissues suggest that this particular design will also expose the soft tissues to potentially damaging localized loads with an associated increased risk of PPUs.

Computational modelling inevitably involves limitations that are associated with the assumptions inherent in the model. First, numerical convergence difficulties compelled us to simulate only $50 \%$ obstruction of the urethra. Additionally, the mechanical properties of the tissues are obtained, as in most computational studies, from animal tissue data. Moreover, the geometry of the penis was developed to represent a nearly symmetrical penis, where an alternative approach would have been to build a subjectspecific model from a 3D set of medical images. Indeed, it should also be recognized 
that relevant patients present with a retracted penis following prostate cancer and this should be considered in future subject-specific models. We did not account for viscoelastic relaxation processes in the distorted tissues, considering that most of these relaxation responses will be completed within several minutes.

The findings that a minor misalignment of the PCC produces an increase in tissue strains and skin stresses has practical implications given the apparent ease of misuse and the natural asymmetries of the human penis. Thus, the issue of adaptability of the clamp to minor misalignments should be a fundamental design requirement. Furthermore, the aforementioned results indicate that, for example, uneven wear of the interface material would have a substantial influence on tissue loads, so durability of the devices also represents a critical design feature. The relevant practical and clinical implications are that PCCs should be tested regularly and their lifetimes limited.

In summary, the modelling study highlights several design features, which should be considered in the evaluation of safety of existing or future designs of PCCs. These are i) low stiffness of materials to enable cushioning ii) adaptability of materials to allow for penis misalignment iii) durability of materials to prevent unequal cushioning iv) absence of any knurls or knobs to reduce high strains/stresses v) absence of compression around the circumference (no cuff) to reduce high strains/stresses vi) contoured design to 'envelop' penis without causing circumferential compression.

We believe this is the first study to systematically model the biomechanical properties of different PCCs. PCCs can be useful devices for men to manage incontinence and may improve quality of life when reconstructive surgery is not advisable or desired. However, 
they may cause discomfort and injure soft tissue which is particularly vulnerable to irreversible damage if loaded for prolonged periods. Using finite element modeling we tested key aspects of current clamps and identified favourable and unfavourable design features. Furthermore the model can be modified using laboratory based in vivo patient data. Combining modelling data with clinical and experimental data should enable the design of a safe, effective and acceptable penile compression clamp for incontinence management. 


\section{References}

[1] Siegel RL, Miller KD, Jemal A. Cancer Statistics, 2015. CA Cancer J Clin 2015; 65: $5-29$.

[2] Stanford JL, Feng Z, Hamilton AS, Gilliland FD, Stephenson RA, Eley JW, Albertsen PC, Harlan LC, Potosky AL. Urinary and sexual function After Radical Prostatectomy for Clinically Localized Prostate Cancer The Prostate Cancer Outcomes Study. JAMA 2000; 283(3): 354-360.

[3] Sacco E, Prayer-Galetti T, Pinto F, Fracalanza S, Betto G, Pagano F, Artibani W. Urinary incontinence after radical prostatectomy: incidence by definition, risk factors and temporal trend in a large series with a long-term follow-up. BJU Int 2006; 97(6): $1234-1241$.

[4] Anderson CA, Omar MI, Campbell SE, Hunter KF, Cody JD, Glazener CMA. Conservative management for post-prostatectomy urinary incontinence. Cochrane Database of Systematic Reviews 2015, Issue 1. Art. No.: CD001843. DOI: 10.1002/14651858.CD001843.pub5

[5] Moore KN, Schieman S, Ackerman T, Dzus HY, Metcalfe JB, Voaklander DC. Assessing comfort, safety, and patient satisfaction with three commonly used penile compression devices. Urology 2004; 63(1):150-154.

[6] Kalra S, Srinivas PR, Manikandan R, Dorairajan LN. Urethral diverticulum: a potential hazard of penile clamp application for male urinary incontinence. BMJ Case Rep 2015. pii: bcr2015209957. doi: 10.1136/bcr-2015-209957. 
[7] Macaulay M, Broadbridge J, Gage H, Williams P, Birch B, Moore KN, Cottenden A, Fader MJ. A trial of devices for urinary incontinence after treatment for prostate cancer. BJU Int 2015; 116(3): 432-442.

[8] Carls C, Ermer-Seltun J, Rolstad BS. Partial thickness wound resulting from use of a penile clamp to control urinary incontinence in a patient with radical prostatectomy [abstract]. In: Symposium on Advanced Wound Care; 2005.

[9] Barnard J, Westenberg AM. The penile clamp: medieval pain or makeshift gain? Neurourol Urodynam 2015; 34(2):115-116.

[10] Linder-Ganz E, Gefen A, Elad D, Chen J. A three-dimensional model of the penis for analysis of tissue stresses during normal and abnormal erection. Ann N Y Acad Sci 2007; 1101: 464-476.

[11] Gefen A, Chen J, Elad D. Stresses in the normal and diabetic human penis following implantation of an inflatable prosthesis. Med Biol Eng Comput 1999; 37(5): $625-631$.

[12] Simpleware ${ }^{\circledR}$ Ltd. ScanIP +FE +NURBS +CAD +FLOW +SOLID +LAPLACE Reference Guide ver. 7.0, 2014. http://www.simpleware.com/software/

[13] FEBio: Finite element for biomechanics, Theory manual ver. 2.3, 2015. http://mrl.sci.utah.edu/software/febio

[14] Maas SA, Ellis BJ, Ateshian GA, Weiss JA. FEBio: Finite Elements for Biomechanics. J Biomech Eng 2012; 134(1): 5-11. 
[15] Linder-Ganz E, Engelberg S, Scheinowitz M, Gefen A. Pressure-time cell death threshold for albino rat skeletal muscles as related to pressure sore biomechanics. $\mathrm{J}$ Biomech 2006; 39(14): 2725-2732.

\section{Figure Captions}

\begin{tabular}{|c|c|}
\hline Figure 1 & $\begin{array}{l}\text { Computational finite element modeling of the penis and penile } \\
\text { compression clamps: (a) A three-dimensional (3D) model of the } \\
\text { penis, with one example of a contoured compression clamp } \\
\text { and corresponding loading configuration. (b) An axial cut } \\
\text { through the penis, showing the skin (S), fat (F), tunica } \\
\text { albuginea (TA), corpus cavernosum (CC), corpus spongiosum } \\
\text { (CS) and urethra (U). (c) The flat, angled, contoured and } \\
\text { contoured with knurl clamps, which were modeled in this work } \\
\text { (left to right). }\end{array}$ \\
\hline Figure 2 & $\begin{array}{l}\text { Distributions of effective tissue stresses in an axial cut through } \\
\text { the penis, while using different penile clamps, at } 50 \% \text { closure } \\
\text { of the urethra. F } 25, \mathbf{F} \mathbf{5 0} \text { and F } 100 \text { are flat clamps with } \\
\text { stiffnesses of } 25,50 \text { and } 100 \mathrm{kPa} \text {; A } 25, \mathbf{A} \mathbf{5 0} \text { and A } 100 \text { are } \\
\text { angled clamps with stiffnesses of } 25,50 \text { and } 100 \mathrm{kPa} \text {; } 25, \mathbf{C} \\
\mathbf{5 0} \text { and C } 100 \text { are contoured clamps with stiffnesses of } 25,50 \\
\text { and } 100 \mathrm{kPa} \text {; Cuff is a cuff-type clamp and Knurl is a } \\
\text { contoured clamp (50 kPa) with knurl (100 kPa). }\end{array}$ \\
\hline Figure 3 & $\begin{array}{l}\text { Comparison of maximal effective and shear strains and } \\
\text { stresses in skin, fat and tunica albuginea, while using different } \\
\text { penile clamps, at } 50 \% \text { closure of the urethra. F } 25, \mathbf{F} 50 \text { and F } \\
100 \text { are flat clamps with stiffnesses of } 25,50 \text { and } 100 \mathrm{kPa} \text {; A } \\
25 \text {, A } 50 \text { and A } 100 \text { are angled clamps with stiffnesses of } 25 \text {, } \\
50 \text { and } 100 \mathrm{kPa} \text {; C } 25, \text { C } 50 \text { and C } 100 \text { are contoured clamps } \\
\text { with stiffnesses of } 25,50 \text { and } 100 \mathrm{kPa} \text {; Cuff is a cuff-type }\end{array}$ \\
\hline
\end{tabular}




\begin{tabular}{|l|l|}
\hline & $\begin{array}{l}\text { clamp and } \mathrm{Knurl} \\
\mathrm{kPa}) .\end{array}$ \\
\hline Figure 4 4 & $\begin{array}{l}\text { Distributions of (a) effective tissue strains and (b) effective } \\
\text { tissue stresses, in an axial cut through the penis, while properly } \\
\text { using a flat } 50 \mathrm{kPa} \text { penile clamp (F 50) and with a } 5^{\circ} \text { tilt of the } \\
\text { bottom half of the clamp (Tilt 50), representing misuse or } \\
\text { malfunction of the clamp. }\end{array}$ \\
\hline
\end{tabular}




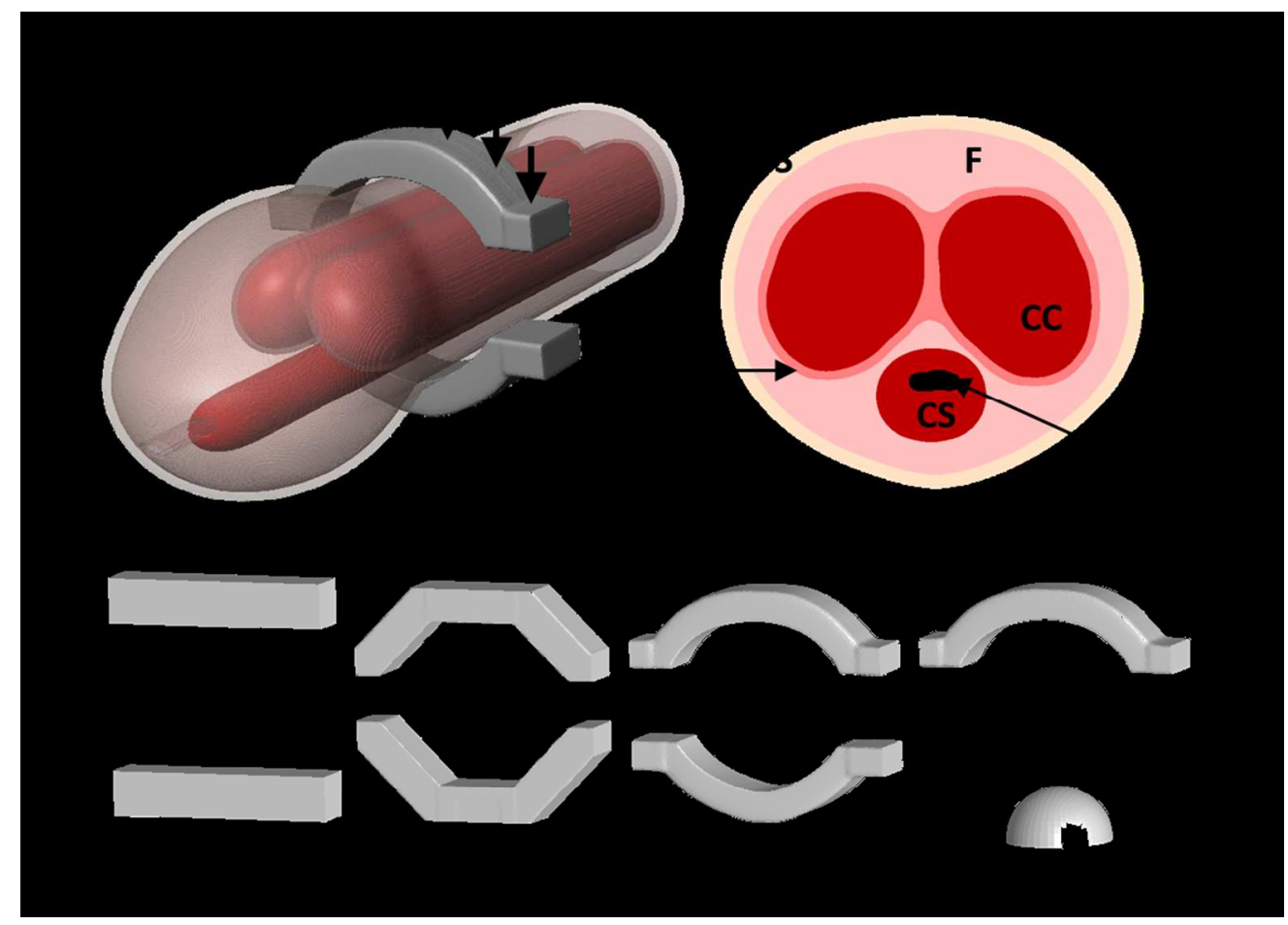

Computational finite element modeling of the penis and penile compression clamps: (a) A three-dimensional

(3D) model of the penis, with one example of a contoured compression clamp and corresponding loading configuration. (b) An axial cut through the penis, showing the skin (S), fat (F), tunica albuginea (TA), corpus cavernosum (CC), corpus spongiosum (CS) and urethra (U). (c) The flat, angled, contoured and contoured with knurl clamps, which were modeled in this work (left to right).

Figure 1a,b Figure 1c $202 \times 144 \mathrm{~mm}(150 \times 150$ DPI $)$ 
Distributions of effective tissue stresses in an axial cut through the penis, while using different penile clamps, at $50 \%$ closure of the urethra. F 25, F 50 and F 100 are flat clamps with stiffnesses of 25,50 and $100 \mathrm{kPa}$; A 25, A 50 and A 100 are angled clamps with stiffnesses of 25, 50 and $100 \mathrm{kPa}$; C 25, C 50 and C 100 are contoured clamps with stiffnesses of 25, 50 and $100 \mathrm{kPa}$; Cuff is a cuff-type clamp and Knurl is a contoured clamp (50 kPa) with knurl (100 kPa).

Figure 2

$198 \times 209 \mathrm{~mm}(150 \times 150 \mathrm{DPI})$ 
Comparison of maximal effective and shear strains and stresses in skin, fat and tunica albuginea, while using different penile clamps, at $50 \%$ closure of the urethra. F 25, F 50 and F 100 are flat clamps with stiffnesses of 25,50 and $100 \mathrm{kPa}$; A 25, A 50 and A 100 are angled clamps with stiffnesses of 25, 50 and $100 \mathrm{kPa}$; C 25, C 50 and C 100 are contoured clamps with stiffnesses of 25, 50 and $100 \mathrm{kPa}$; Cuff is a cufftype clamp and Knurl is a contoured clamp $(50 \mathrm{kPa})$ with knurl $(100 \mathrm{kPa})$.

\section{Figure 3}

$189 \times 189 \mathrm{~mm}(150 \times 150 \mathrm{DPI})$ 
Distributions of (a) effective tissue strains and (b) effective tissue stresses, in an axial cut through the penis, while properly using a flat $50 \mathrm{kPa}$ penile clamp ( $\mathrm{F} 50)$ and with a $5^{\circ}$ tilt of the bottom half of the clamp (Tilt $50)$, representing misuse or malfunction of the clamp.

\section{Figure 4}

$191 \times 169 \mathrm{~mm}(150 \times 150 \mathrm{DPI})$ 
Table 1: Variants of the finite element modeling.

\begin{tabular}{cccc}
\hline Model variant & Clamp geometry & Stiffness [kPa] & $\begin{array}{c}\text { Final contact area } \\
\text { with skin [mm }{ }^{2}\end{array}$ \\
\hline F 25 & Flat & 25 & 387 \\
\hline F 50 & Flat & 50 & 247 \\
\hline F 100 & Flat & 100 & 191 \\
\hline A 25 & Angled & 25 & 639 \\
\hline A 50 & Angled & 50 & 618 \\
\hline A 100 & Angled & 100 & 607 \\
\hline C 25 & Contoured & 25 & 769 \\
\hline C 50 & Contoured & 50 & 769 \\
\hline C 100 & Contoured & 100 & 769 \\
\hline Cuff & Cuff-type & none & N/A \\
\hline Knurl & Contoured & 50,100 & 296 \\
\hline Tilt 50 & with knurl & 50 & 522 \\
\hline
\end{tabular}

